\title{
Withdraw life sustaining treatment and organ donation
}

\author{
Seungyre Jeong ${ }^{1}$, Younghwan Hwang ${ }^{1}$, Minyoung Chu ${ }^{1}$, Ohhyuk Yun ${ }^{1}$, Youngsoon Jeong ${ }^{1}$, Jeongrim Lee ${ }^{1}$, Won-Hyun Cho ${ }^{2}$ \\ ${ }^{1}$ Department of Coordinator, Korea Organ Donation Agency, Seoul, Korea \\ ${ }^{2}$ Korea Organ Donation Agency, Seoul, Korea
}

Background: According to the statistics of the National Agency for Management of Life-Sustaining Treatment, 85,000 end of life care patients have been withdrawn life sustaining treatment over two years since the implementation of law in February 2018. However, according to annual report of Korea Organ Donation Agency (KODA), there were a considerable number of patients who requested to withdraw life sustaining treatment among the referred potential brain death to the call center of KODA. Their family consent rate was lower than the group that didn't mentioned about interrupting the end of life care.

Methods: The study was designed using the referred cases of potential brain death to KODA during 2018 and 2019. Demographic findings of requesting withdrawn life sustaining treatment among the referred cases and their result of donation progress in each step were evaluated.

Results: Within the research period, there were a total of 4,910 cases $(2,426$ in 2018, 2,484 in 2019) of brain death, and among them, 569 (193 in 2018 and 376 in 2019) cases requested to withdraw life sustaining treatment. This means a $94.8 \%$ increase in requesting family compared to the previous year. In terms of gender, $63 \%$ of the cases were male, and the most common age group was the fifties (23.4\%). Among 569 requested donors, 475 (83.5\%) were appropriate for organ donation, out of which, 394 (82.9\%) families were interviewed about organ donation, and accepted organ donation in 45 cases (9.5\%). Only 29 cases (6.1\%) were succeeded in organ procurement.

Conclusions: It is estimated that there will be more families who wish to withdraw life sustaining treatment among potential brain death referred to KODA for organ donation in the future. Therefore, in order to activate organ donations, we need a system revision about end of life care process, first to the donor coordinator and then explain all the options by that coordinator. 